\section{Studien: Charta gegen Altersdiskriminierung}

— Eine internationale Vereinigung von Geriatern moniert eine Altersdiskriminierung bei klinischen Untersuchungen. Das von der EU finanzierte Projekt mit der Bezeichnung PREDICT erinnert daran, dass z.B. in der Onkologie gerade geriatrische Patienten das Gros der chemotherapierten Patienten bilden - dass sich aber die Ergebnisse klinischer Studien, gewonnen anhand eines eher jüngeren Teilnehmerfelds, nicht immer für ältere Menschen extrapolieren lassen. PREDICT will das Ausmaß beurteilen, in dem ältere Menschen von klinischen Tests ausgeschlossen sind und mögliche Lösungen vorschlagen. Koordiniert wird das Projekt [www.predicteu.org] vom britischen Medical Economics and Research Centre. Aus Deutschland beteiligt sich leider keine Institution.

ro

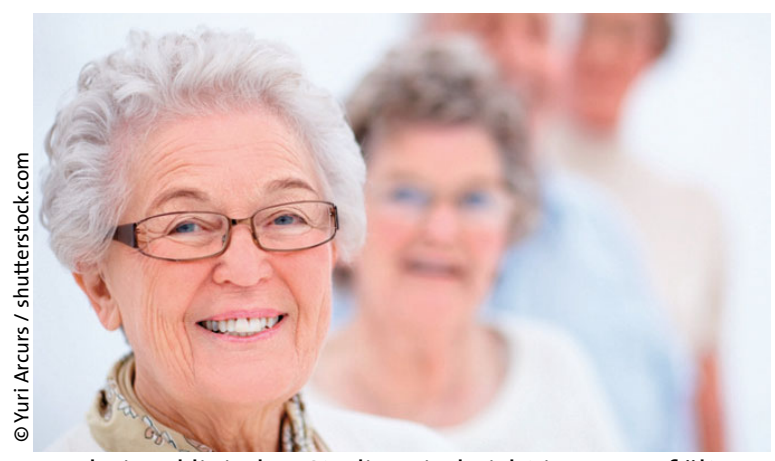

Ergebnisse klinischer Studien sind nicht immer auf ältere Menschen anwendbar.

\section{Arzneimittel \\ Krankenkassen wollen sparen}

- Bei einem Treffen zwischen dem Verband der Gesetzlichen Krankenkassen und Bundesgesundheitsminister Rösler wurden von den Kassen Vorschläge unterbreitet, wie Kosten eingespart werden könnten. Der Vizevorsitzende des Verbandes, Johann-Magnus von Stackelberg, erklärte nach dem Treffen gegenüber der Frankfurter Allgemeinen Zeitung:,,Wir haben kurzfristige Maßnahmen unterbreitet, um weitere Zusatzbeiträge zu verhindern."

Die Krankenkassen wollen eine Halbierung der Mehrwertsteuer auf Arzneimittel; dies bedeutet eine Ersparnis von rund 2,8 Mrd. Euro. Insgesamt belaufen sich die Sparvorschläge auf über 4 Mrd. Euro. Dabei waren für den Gesundheitsminister vor allem langfristige Maßnahmen bei innovativen Arzneimitteln wichtig. Diese gelten laut Rösler als Kostentreiber. Hier laufen auch Gespräche mit der Pharmaindustrie.

Mittlerweile hat Arbeitsministerin von der Leyen ihre Ankündigung zurückgenommen, Hartz-IV-Empfänger generell von Zusatzbeiträgen zu befreien beziehungsweise diese Beiträge von den Arbeitsagenturen übernehmen zu lassen. Die Kosten für die pauschale Übernahme der Zusatzbeiträge hätten 300 Mio. Euro betragen. Für Härtefälle, so der stellvertretende Fraktionsvorsitzende der Union, Johannes Singhammer, gebe es bereits jetzt Ausnahmen.

\section{Stationäre Versorgung Lebensgefahr in vollen Kliniken?}

— In voll belegten Krankenhäusern ist das Risiko zu sterben erhöht, zeigte eine Studie der University of Michigan mit 166.00o Patienten, die im Laufe von drei Jahren in verschiedenen Kliniken aufgenommen worden waren [Medical Care, 2010;48(3): 224DOI:10.1097/ MLR.obo13e3181c162co]. Bei einer Bettenbelegung von über $80 \%$ stieg die Mortalität der Patienten um 5,6\%. Ein um 7,5\% erhöhtes Sterberisiko ergab sich für Patienten, die am Wochenende aufgenommen wurden, und auch in der Influenzasaison war das Risiko deutlich (um 11,7\%) erhöht. Als wesentlicher Faktor, der die Sterblichkeit senkt, wurde die Zahl der Krankenschwestern identifiziert: Je mehr Schwestern in der Klinik tätig waren, desto günstiger war die Prognose der Patienten. Wie sich die Arztdichte auf die Überlebenschancen auswirkt, wurde nicht geprüft.

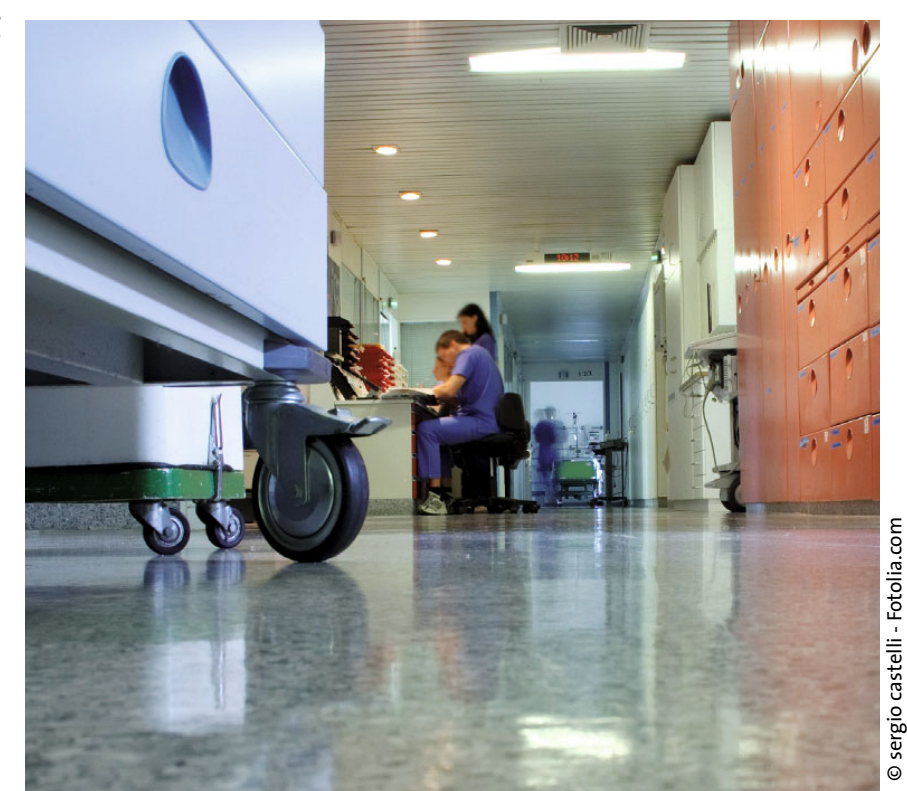

In vollen Kliniken kommt man schneller unter die Räder ... 